\title{
NUCLEAR MAGNETIC RELAXATION DISPERSION STUDY OF HUMAN SERUM PROTEINS SOLUTIONS
}

\author{
K.J. OLSZEWSKI \\ Department of Physics, Agricultural University in Poznań \\ Wojska Polskiego 38/42, 60-637 Poznań, Poland \\ (Received January 28, 1992; in final form May 14, 1992)
}

\begin{abstract}
Nuclear magnetic relaxation dispersion profiles in the Larmor frequency range of $0.02-50 \mathrm{MII}$ and $277 \mathrm{~K}$ has been studied for human serum proteins: albumin, $\gamma$-globulins and $\alpha, \beta$-globulins in aqueous solutions 3-14 per cent by weight of protein. The linear concentration dependences have been found both for the longitudal relaxation rates for water protons and the dispersive and non-dispersive parts of relaxation profiles. The frequency dependent relaxivities have been calculated, the hydrodynamic effect for the albumin macromolecule Brownian rotational correlation time has been observed and analyzed. The importance of molecular weight of protein for the relaxation processes and its influence for magnetic resonance imaging contrast was discussed.
\end{abstract}

PACS numbers: $76.90 .+\mathrm{d}, 87.45 .-\mathrm{k}$

\section{Introduction}

From the early sixties nuclear magnetic relaxation processes of water molecules in aqueous solutions of diamagnetic proteins [1] have often been examined analyzing the water-proton interaction in biological systems [2], particularly for understanding the mechanism of magnetic resonance imaging (MRI) contrast [3]. The study of serum proteins is particulary interesting here [4-7], especially by means of field cycling technique [8], which allows to examine the relaxation process in the wide range of proton Larmor frequencies, then for different magnetic fields. Using the latter or similar experimental methods, many examinations of diamagnetic protein systems have been performed, especially for different globular protcins [9-16].

The plot of the relaxation rates (inversion of the spin-lattice relaxation time $\left.T_{1}\right)$ vs. the Larmor frequency is often called the nuclear magnetic relaxation dispersion (NMRD) profile. As it was shown by Koenig and co-workers [10] NMRD 
profiles can be described by the formula based on the Cole-Cole expression [17] in the following form:

$$
R_{1}=R_{1 \mathrm{w}}+D+\frac{A\left[1+\left(\nu / \nu_{\mathrm{c}}\right)^{\beta / 2} \cos (\pi \beta / 4)\right]}{1+2\left(\nu / \nu_{\mathrm{c}}\right)^{\beta / 2} \cos (\pi \beta / 4)+\left(\nu / \nu_{\mathrm{c}}\right)^{\beta}}
$$

where $R_{1 \mathrm{w}}+D$ represents the non-dispersive part of the profile, $A$ is the dispersive part, $\nu_{\mathrm{c}}$ is the inflection frequency which corresponds to the correlation time for Brownian reorientation of macromolecule $\tau_{R}=\sqrt{3} /\left(2 \pi \nu_{c}\right)$ and $\beta$ is the steepness of the inflection step. For $\beta=2$ the expression (1) reduces to the constant with the Lorenzian term:

$$
R_{1}=R_{1 \mathrm{w}}+D+\frac{A}{1+\left(\nu / \nu_{\mathrm{c}}\right)^{2}}
$$

which is formally similar to the simplification of the Bloembergen, Purcell and Pound formula describing the relaxation rate under dipolar proton-proton interaction [18].

According to Koenig, the expression (1) should be regarded as a heuristic equation and does not have a priori validity [10]. Nonetheless, it has been experimentally noticed that the inflection frequency is closely correlated to the molecular weight of the macromolecule. On the other hand, the non-dispersive term seems to be associated with water molecules exchanging between the bulk water and the hydration shell of the macromolecule. The mechanism underlying $A$ term is somehow elusive. Considering the fact that this term is influenced by the motion of protein, Koenig suggests that the angular momentum of the macromolecule can be transferred to the solvent water molecules by the long range hydrodynamical interaction. This "slosh effect" causes all of the solvent molecules, excluding those from the first hydration layer, to have a small component of their thermal motion that follows the Brownian motion of the protein macromolecule [19].

The aim of the present work was to examine NMRD profiles for protons in aqueous solutions of fundamental components of human serum: albumin, $\alpha, \beta$-globulins and $\gamma$-globulins in the wide range of protein contents, ranging from lower than the physiological ones, up to over a dozen or so per cent by weight. The results were analyzed in terms of the concepts given by Koenig and co-workers. The correlation between the experimental relaxation rates or phenomenological parameters in Eq. (1) and protein concentration was examined and discussed.

\section{Material and methods}

The samples were prepared from commercial crystalized and liophylized human serum proteins produced by Sigma, i.e. albumin (No. A-9511), $\gamma$-globulins (No. G-4386) and globulins (No. G-3637- the mixture of $\alpha_{2^{-}}$and $\beta_{1}$-globulins from Cohn fraction IV, 4), by diluting in $0.05 \mathrm{M}$ saline Tris buffer pII 7.3. The range of applied proteins concentrations was changed from 3 per cent by weight (pcw) up to more than $14 \mathrm{pcw}$. After preparations the samples were stabilized for $24 \mathrm{~h}$ at room temperature, during the NMR measurements they were stored at 4 centigrade in the refrigerator. The samples were not degassed. 
NMRD profiles were recorded by using the IBM Research Relaxometeres and the experimental technique proposed by Koenig and Brown [8]. The relaxation rate $R_{1}=T_{1}^{-1}$ for a given proton Larmor frequency was determined from the dependence of spin echo amplitudes vs. applied measure-field times by the linear regression method. For all the studied samples spin echo delays were monoexponential, the experimental error was in the range of 3-5 per cent.

The measurements were taken at $4^{\circ} \mathrm{C}(277 \mathrm{~K})$, the temperature was stabilized by the flow of liquid perfluoroethylen.

NMRD profiles were calculated using the formula (1) by the computer fit of the experimental data with the Minuit program from CERN program library.

During all the experiments the reproducibility and accuracy of the measurements were carefully controlled using the standard $\mathrm{MnCl}_{2}$ doped sample with a known relaxation rate.

\section{Results and discussion}

The recorded typical NMRD profiles for the chosen human serum albumin (IISA) concentrations in the range of 3-14.2 pcw are presented in Fig. 1.

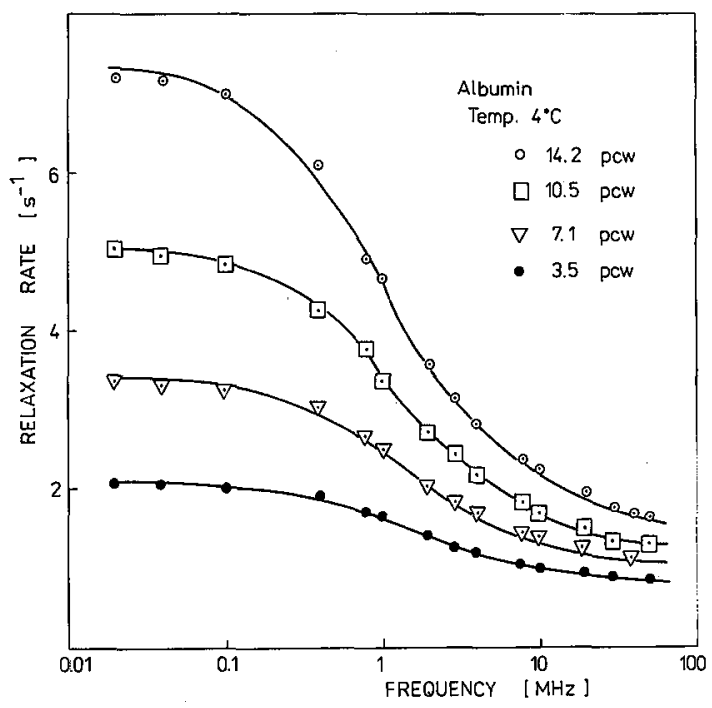

Fig. 1. The typical NMRD profiles for human serum albumin solutions in Tris bufter pII 7.3. Protein concentrations expressed in per cent by weight (pcw). Relaxation rates were recorded at $4^{\circ} \mathrm{C}(277 \mathrm{~K})$, the solid lines were calculated using Eq. (1).

The following figures present NMRD profiles for solutions of $\gamma$-globulins 4-14.2 pcw (Fig. 2), and other globulins 4-14 pcw (Fig. 3) at the same temperature of $4^{\circ} \mathrm{C}$. 

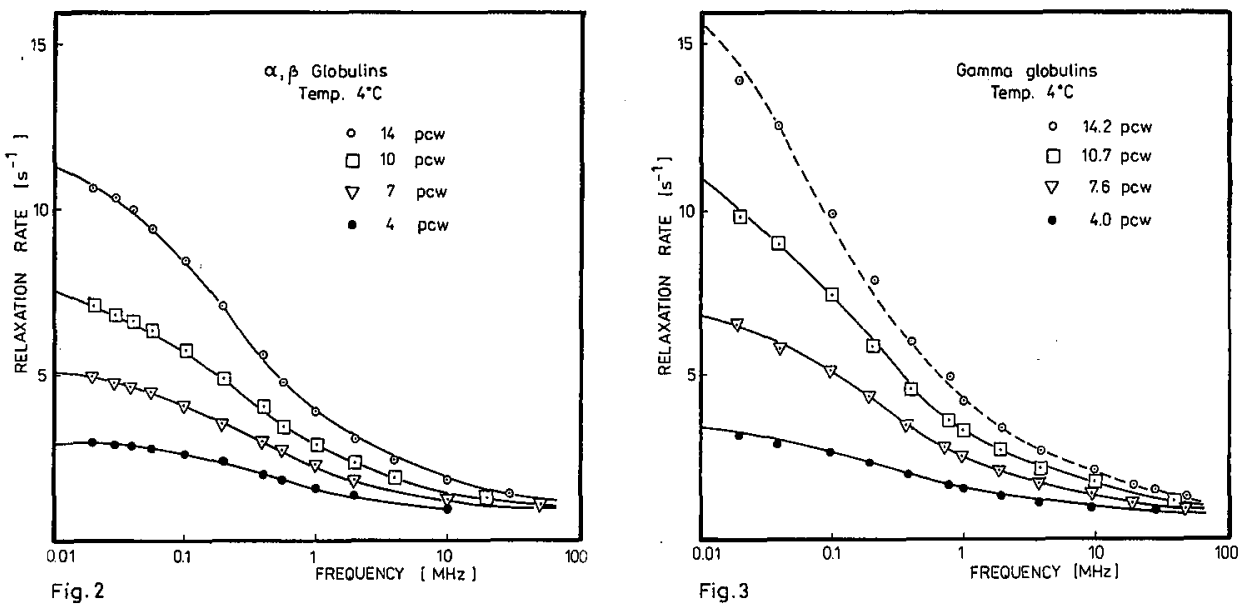

Fig. 2. NMRD profiles for human serum $\alpha, \beta$-globulins (Cohn fraction IV, 4) solutions in Tris buffer pH 7.3. The details as above.

Fig. 3 NMRD profiles for human serum $\gamma$-globulins solutions in Tris bufter pII 7.3. The details as above.

The fitting parameters determined from the expression (1) are listed in Table I for all examined proteins and their concentrations separately.

Additionally, the relaxation rates for the solvent were also measured. A slight frequency dependence was observed, the relaxation rate in the higher fields was $0.64 \mathrm{~s}^{-1}$ and increased in the lowest fields up to $0.74 \mathrm{~s}^{-1}$.

For all the examined proteins, the relaxation rates at the given proton Larmor frequency increased with the protein content. According to Koenig and Schilinger [9] this dependence can be described by the linear function of the protein molality in the following form:

$$
R_{1}=\frac{N R}{1-V}+R_{1 \mathrm{w}}
$$

where $R_{1}$ is the measured relaxation rate at the given field, $R_{1 \mathrm{w}}$ is the relaxation rate for solvent protons, $N$ is the molar concentration of the given protein, and $V$ is the volume fraction. The frequency dependent quantity of $R$ that corresponds to the slope of this dependence, the so-called relaxivity, expresses the relaxation increments brought by one mole of the given protein. Using Eq. (3) the values of relaxivities were determined for the given proton Larmor frequencies, and then, the Colc-Cole fit was performed for all the studied proteins. The calculated parameters are listed in Table II.

It can be noticed that non-dispersive terms of the relaxivity for the examined proteins are slight and not very distinguishable. On the other hand, the large difference exists for dispersive parts. In the lowest field they are $2.4 \mathrm{mM} \mathrm{s}^{-1}$ for IISA, $7.6 \mathrm{mM} \mathrm{s}^{-1}$ for globulins and even $14.1 \mathrm{mM} \mathrm{s}^{-1}$ for $\gamma$-globulins. This suggests that globulins, particularly $\gamma$-globulins with the molecular weight of $160 \mathrm{kD}$, are more effective in influencing the relaxation rate of the protein solution comparing to the relatively small (molecular weight of $60 \mathrm{kD}$ ) IISA molecule. Then the possibility of 
TABLE I

The fitted parameters from the Cole-Cole formula (1) for the studied human serum proteins solutions in Tris buffer pH 7.3 and at the temperature of $4^{\circ} \mathrm{C} . D$ represents the non-dispersive part, $A$ - the dispersive part, $\nu_{c}-$ infection frequency, $\beta-$ steepness of the dispersion step.

\begin{tabular}{c|r|r|r|r|c|c}
\hline \hline Protein & $\begin{array}{c}\text { total } \\
\text { content } \\
{[\mathrm{pcw}]}\end{array}$ & $\begin{array}{c}\text { molality } \\
{\left[\mathrm{mM} / \mathrm{kg}^{2}\right]}\end{array}$ & $\begin{array}{c}\mathrm{D} \\
{\left[\mathrm{s}^{-1}\right]}\end{array}$ & $\begin{array}{c}\mathrm{A} \\
{\left[\mathrm{s}^{-1}\right]}\end{array}$ & $\begin{array}{c}\nu_{\mathrm{c}} \\
{[\mathrm{MIIz}]}\end{array}$ & $\beta$ \\
\hline \multirow{5}{*}{ Albumin } & 14.20 & 2.40 & 1.49 & 6.08 & 1.07 & 1.39 \\
& 14.16 & 2.39 & 1.60 & 5.76 & 0.99 & 1.45 \\
& 11.90 & 1.96 & 1.42 & 4.18 & 1.11 & 1.54 \\
& 10.50 & 1.71 & 1.17 & 4.01 & 1.28 & 1.40 \\
& 7.10 & 1.11 & 0.98 & 2.49 & 1.52 & 1.40 \\
& 7.10 & 1.11 & 1.12 & 2.36 & 1.41 & 1.54 \\
& 3.50 & 0.52 & 0.81 & 1.30 & 1.69 & 1.44 \\
& 3.00 & 0.45 & 0.75 & 1.10 & 1.79 & 1.34 \\
\hline \multirow{5}{*}{$\beta$-globulins } & 14.00 & 1.38 & 0.89 & 12.20 & 0.20 & 1.11 \\
& 10.00 & 0.94 & 0.81 & 7.58 & 0.25 & 1.13 \\
& 7.00 & 0.64 & 0.76 & 4.69 & 0.31 & 1.16 \\
& 4.00 & 0.35 & 0.73 & 2.49 & 0.34 & 1.20 \\
\hline & 14.17 & 1.03 & 0.73 & 21.00 & 0.06 & 0.96 \\
& 10.71 & 0.75 & 0.76 & 12.90 & 0.10 & 1.02 \\
& 7.63 & 0.52 & 0.63 & 7.85 & 0.14 & 0.92 \\
& 4.03 & 0.26 & 0.70 & 2.96 & 0.25 & 1.10
\end{tabular}

distinguishing the protein by its characteristic relaxation rate (the MRI contrast) increases with lowering of the frequency and magnetic field.

The calculated parameters expressing the non-dispersive and dispersive terms

TABLE II

The fitted parametres from the Cole-Cole formula (1) for relaxivities of human scrum proteins. The details as before.

\begin{tabular}{c|c|c|c|c}
\hline \hline Protein & $\begin{array}{c}\mathrm{D} \\
{\left[\mathrm{mM} \mathrm{s}^{-1}\right]}\end{array}$ & $\begin{array}{c}A \\
{\left[\mathrm{mM} \mathrm{s}^{-1}\right]}\end{array}$ & $\begin{array}{c}\nu_{c} \\
{[\mathrm{MIIz}]}\end{array}$ & $\beta$ \\
\hline $\begin{array}{c}\text { Albumin } \\
\alpha, \beta \text {-globulins }\end{array}$ & 0.40 & 2.08 & 1.00 & 1.57 \\
$\gamma$-globulins & 0.13 & 10.19 & 0.14 & 1.05 \\
& 0.26 & 19.37 & 0.09 & 1.06
\end{tabular}


in the formula (1) depended also on protein concentration. The non-dispersive term can be expressed as a linear function of the protein content, what reflects the increase in the bound water fraction while the total protein content increases. The extrapolation to null protein content gives the value very close to $0.64 \mathrm{~s}^{-1}$ corresponding to the relaxation rate of pure solvent. The dispersive part also seems to depend linearly on protein concentrations, however, some diversion can be seen for the samples containing more than 12 per cent of IISA. It seems to be in relation to the observed nonlinear effects above the "critical volume" [20,21], attributed due to macromolecular aggregation at the higher proteins contents, or, what has been suggested recently [22], to the depressing of the macromolecular tumbling and the increase of the relaxation contribution from translational surface diffusion of water in the hydration shell.

The inflection frequencies are closely correlated with the molecular weight of the studied protein and change slightly while protein concentration increases. Similar effects were observed for hemoglobin solutions [23] and were explained as the increase of the hydrodynamical interaction of the macromolecule with their neighbours. In this approach, the free reorientation of the macromolecule at infinite dilution is restricted when other macromolecules appear closer and closer while the protein contents increase. Assuming that the macromolecule is positioned in spherical cage created by the neighbours, the rotational correlation time $\tau_{R}$ is changed by changing the local micro viscosity of the solvent as follows:

$$
\tau_{R}=\frac{\tau_{0}}{1-\left(\frac{r}{D-r}\right)^{3}},
$$

where $\tau_{0}$ is the correlation time at dilute limit, $D$ is the mean distance between the macromolecules, and $r$ is the hydrodynamical radius of the protein (assuming spherical) with a single hydration layer. This approximation gives for IISA data the value of $\tau_{0}=1.6 \times 10^{-7} \mathrm{~s}$ and $R=41 \AA$. The value of the Brownian rotational correlation time at the dilute limit is however greater than those from EPR spin label experiments [24] or dielectric study [25]. It is also greater than the correlation time obtained from the simple Einstein-Stokes formula, which is of $3.5 \times 10^{-8} \mathrm{~s}$. On the other hand, the estimated parameter for the hydrodynamical radius of the HSA macromolecule is also distinctly different from those calculated from partial specific volume data [26], where the quantity of $27 \AA$ is obtained. So, one can speculate that for the assumed hydrodynamical concepts it is necessary to consider the reorienting macromolecule-hydration shell complex as a much larger as it can be suggested from the structural data of the HSA molecule [27].

Finally, the values for $\beta$ parameters corresponding to the steepness of the inflection step are, in case of IISA solutions, similar for all the studied concentrations and the mean value of $\beta$ is equal to 1.3. This parameter is difficult to interpretate owing to the fact that the Cole-Cole formula (1) treats this parameter formally as the width of the correlation time distribution, which is not considered or analyzed in the Koenig hydrodynamical model. It can only be asserted that the fitted $\beta$ value is significantly different from $\beta=2$. So, for the studied case the simple two-state model establishing only free and bound water is experimentally excluded here. 


\section{Conclusions}

1. NMRD profiles for serum proteins solutions are similar for those for diamagnetic protein solutions and the measured relaxation rates appear to be strongly dependent on proton Larmor frequency corresponding to the measure-field value.

2. For the given proton Larmor frequency the relaxation rate depends linearly on protein content. This allows to determine the relaxivities for the given protein. The relaxivity plots also prove to be frequency dependent and seem to correspond to the molecular weight of the protein.

3. NMRD profiles can be approximated by the formula proposed by Koenig based on the Cole-Cole dispersion formula, the parameters of this fit (excluding $\beta)$ are also concentration dependent.

4. The main factor determining the relaxation rates (apart from protein content) is the molecular weight of the protein influencing both the dispersion term of the relaxation rate or the inflection frequency and the relaxivity. The effect of changing in the hydration layer (bound water fraction), however observed, seems to be less important.

5. In consequence, the possibility of distinguishing the protein using its characteristic relaxation rate (the contrast effect) increases with decreasing in field value, where the proton Larmor frequency is closer to those of the protein reorientational motion.

\section{Acknowledgements}

The author wishes to thank Dr.R.N. Muller who has helped to improve this work with his friendly assistance, suggestions and goodwill. I am also indebted to Mr. F. Maton and Dr. H.W. Fisher for the help in the experimental work. Finally, the financial support from the University of Mons and Prof. Y. van Haverbake's interest in it, is gratefully acknowledged.

\section{References}

[1] O.K. Daszkiewicz, J.W. Ifennel, B. Lubas, T.W. Szczepkowski, Nature 200, 1006 (1963).

[2] R.G. Bryant, W.M. Shirley, Am. Chem. Soc. Symp. Ser. 127, 147 (1980).

[3] P. Mansfield, P.G. Morris, NMR Imaging in Biomedicine, Academic Press, New York 1982.

[1] J. Oakes, J. Chem. Soc. Faraday Trans. 72, 216 (1976).

[5] M.F. Fuller, W.S. Brey, J. Biol. Chem. 243, 274 (1968).

[6] J. Gallicr, P. Rivet, J. de Certains, Biochim. Biophys. Acta 915, 1 (1987).

[7] R.S. Menon, P.S. Allen, Biophys. J. 57, 389 (1990).

[8] S.H. Koenig, R.D. Brown III, in NMR Spectroscopy of Cells and Organisms, Ed. R. Gupta, CRC Press, Boca Raton 1987, p. 75.

[9] S.H. Koenig, J. Schillinger, J. Biol. Chem. 244, 3283 (1969).

[10] K. Hallenga, S.II. Koenig, Biochemislry 15, 4255 (1976). 
[11] I. Andersson, W. Maret, M.Zeppezauer, R.D. Brown III, S.II. Koenig, Biochemistry 20, 3424 (1981).

[12] S.H. Koenig, R.D. Brown III. J.F. Gibson, R.J. Ward, T.J. Peters, Mag. Reson. Med. 3, 755 (1986).

[13] J.I. Bertini, C. Luchinati, R.D. Brown III, S.H. Koenig, J. Am. Chem. Soc. III, 3532 (1989).

[14] H.H. Raeymaekers, H. Eisendrath, A. Varbeken, Y. van IIaverbeke, R.N. Muller, J. Magn. Reson. 85, 421 (1989).

[15] L. Grösch, F. Noack, Biochim. Biophys. Acta 453, 218 (1976).

[16] R. Kimmich, W. Nusser, T. Gneiting, Colloids Surf. 45, 283 (1990).

[17] K.S. Cole, R.H. Cole, J. Chem. Phys. 9, 341 (1941).

[18] N. Bloembergen, E.M. Purcell, R.V. Pound, Phys. Rev. 73, 679 (1948).

[19] S.H. Koenig, Am. Chem. Soc. Symp. Ser. 127, 157 (1980).

[20] R. Giordano, A. Salleo, S. Salleo, F. Wanderlingh, Phys. Lelt. A 70, 64 (1979).

[21] B. Blicharska, Acta Phys. Pol. A70, 121 (1986).

[22] R. Kimmich, T. Gneiting, K. Kolitschke, G. Schnur, Biophys. J. 58, 1183 (1990).

[23] T.R. Lindstrom, S.H. Kocnig, J. Magn. Reson. 15, 344 (1974).

[24] S. Capistrano, F. Saccheti, Phys. Rev. A 33, 745 (1986).

[25] J.T. Edsall, in The Prolein I, Ed. II. Neurath, Academic Press, New York 1953, p. 702 .

[26] J.L. Oncley, G. Scatchard, A. Brown, J. Phys. Colloid Chem. 51, 184 (1947).

[27] T.E. Creightron, Proteins Slruclures and Molecular Properties, W.II. Freeman, New York 1983, p. 184. 